\title{
Effects of Polyelectrolyte Chain Stiffness, Charge Mobility, and Charge Sequences on Binding to Proteins and Micelles
}

\author{
Christy L. Cooper, Ann Goulding, A. Basak Kayitmazer, Serge Ulrich, ${ }^{\ddagger}$ Serge Stoll, ${ }^{\ddagger}$ \\ Sibel Turksen, Shin-ichi Yusa, $§$ Anil Kumar, and Paul L. Dubin*,† \\ Department of Chemistry, Indiana Purdue University, Indianapolis, Indiana 46202
}

Received August 18, 2005; Revised Manuscript Received December 26, 2005

\begin{abstract}
The binding affinities of polyanions for bovine serum albumin in $\mathrm{NaCl}$ solutions from $I=0.01-0.6 \mathrm{M}$, were evaluated on the basis of the $\mathrm{pH}$ at the point of incipient binding, converting each such $\mathrm{pH}_{\mathrm{c}}$ value into a critical protein charge $Z_{\mathrm{c}}$. Analogous values of critical charge for mixed micelles were obtained as the cationic surfactant mole fraction $Y_{\mathrm{c}}$. The data were well fitted as $Y_{\mathrm{c}}$ or $Z_{\mathrm{c}}=K I^{a}$, and values of $K$ and $a$ were considered as a function of normalized polymer charge densities $(\tau)$, charge mobility, and chain stiffness. Binding increased with chain flexibility and charge mobility, as expected from simulations and theory. Complex effects of $\tau$ were related to intrapolyanion repulsions within micelle-bound loops (seen in the simulations) or negative protein domainpolyanion repulsions. The linearity of $Z_{\mathrm{c}}$ with $\sqrt{ } I$ at $I<0.3 \mathrm{M}$ was explained by using protein electrostatic images, showing that $Z_{\mathrm{c}}$ at $I<0.3 \mathrm{M}$ depends on a single positive "patch"; the appearance of multiple positive domains $I>0.3 \mathrm{M}$ (lower $\mathrm{pH}_{\mathrm{c}}$ ) disrupts this simple behavior.
\end{abstract}

\section{Introduction}

The electrostatic interaction of polyelectrolytes and proteins is relevant to many physiological processes and to a number of applications. As an example of in vivo phenomena, the glycosaminoglycans (GAGs) heparin and heparan sulfate interact with the protein antithrombin to regulate the blood coagulation cascade. ${ }^{1}$ Similarly, the GAG hyaluronic acid forms ternary complexes with cartilage link protein and the proteoglycan aggrecan to provide joint tissue with its load-bearing properties. ${ }^{2}$ With regard to applications, protein-polyelectrolyte interactions are central to protein separations, $, 3,4$ biosensor development, ${ }^{5}$ and enzyme immobilization. ${ }^{6}$ The general subject of proteinpolyelectrolyte interactions has been recently reviewed by Cooper et al. ${ }^{7}$

At least in part, protein-polyelectrolyte interactions may be considered a subset of polyelectrolyte-colloid interactions, although the issue is complicated by the question of "specific" vs "nonspecific" interactions in biological cognate pairs. Nevertheless, the fundamental relations anticipated on the basis of long-range electrostatic effects cannot be held in abeyance even for biologically co-evolved systems. One consequence of these interactions is the central role of three variables: the colloid surface charge density $\sigma$, the polyelectrolyte linear charge density $\xi$, and the ionic strength, typically embodied in the Debye-Hückel parameter $\kappa$. An important consequence of theory ${ }^{8,9}$ is the finding of a transition from bound to unbound state with a change in any of those three variables, so that the critical conditions for binding at constant temperature could be

* To whom correspondence should be addressed. E-mail: dubin@ chem.umass.edu.

Current address: Department of Chemistry, University of Massachusetts, Amherst, Massachusetts 01003.

$\doteqdot$ Department of Inorganic, Analytical, and Applied Chemistry, University of Geneva, Sciences II 30, Quai Ernest-Ansermet 1211, Geneva 4, Switzerland. E-mail: serge.ulrich@cabe.unige.ch (S.U.).

$\S$ Department of Materials Science and Chemistry, Graduate School of Engineering, University of Hyogo, 2167 Shosha, Himeji, Hyogo 671-2201, Japan. expressed as

$$
\sigma_{\mathrm{c}} \xi \sim \kappa^{b}
$$

a result initially obtained for polyelectrolyte adsorption onto flat surfaces, but subsequently found for spherical colloids as well,,${ }^{9,10}$ with values of $b$ ranging from 0.5 to $3 .^{8-11}$ This result has been supported by experiments with various polyelectrolytes interacting with oppositely charged micelles ${ }^{12-17}$ and large dendrimers (smaller dendrimers behaving more like large counterions). ${ }^{14,18}$ Still, discrepancies between the experimental systems and the theoretical model must be noted. With micelles, the surface charge density is controlled by the mole fraction of ionic surfactant $(Y)$ or by the degree of ionization of acidic or basic surfactant headgroups ( $\alpha$ or $\beta$, respectively), and one may in fact observe a critical value for $Y$ (or $\alpha$ or $\beta$ ) at the onset of complex formation. ${ }^{12,13,19}$ However, equating $Y$ (or $\alpha$ or $\beta$ ) with $\sigma$ presumes, among other things, that the distribution of ionic surfactant headgroups is uniform and that these headgroups can be considered to define a "surface". Once such assumptions are made, it is possible in the case of micelles with surfactant headgroups amenable to potentiometric titration, to determine $\left(\psi_{0}\right)_{\mathrm{c}}$, the surface potential at critical binding conditions. ${ }^{20}$ From this value, in conjunction with micelle radius and ionic strength, one may obtain $\sigma_{\mathrm{c}}$ by using the Poisson-Boltzmann equation in approximate analytical form or via numerical solutions, ${ }^{21}$ and so rationalize experiment in terms of eq 1.

The situation is evidently more complicated for proteins, where the simple observation of binding "on the wrong side of the isoelectric point" clearly indicates that polyelectrolytes may interact with a charged domain that may be opposite in sign to the net protein charge. For a half-dozen polyelectrolyte-protein pairs, it was shown that this situation gives rise to maxima in the binding affinity as a function of ionic strength $I$, with $I_{\max }$ corresponding to Debye lengths close to the protein radius. ${ }^{22}$ The exact location of this electrostatic binding site is not a priori known, nor is it constant over the entire $I$ range, although Grymonpré et al. suggested criteria by which it might be 
identified. ${ }^{23}$ The question of a positive electrostatic binding site encompassed within a globally negative protein is certainly of biological interest, as anionic biopolyelectrolytes such as GAGs do bind to proteins with $\mathrm{p} I<7$, i.e., proteins with net negative charge under physiological conditions. ${ }^{24}$ But the nonuniformity of protein charge distributions obscures the relevance of eq 1 to polyelectrolyte-protein interactions, despite the tantalizing finding for at least one polyelectrolyte-protein system, of a linear dependence on $I^{1 / 2}$ of the net protein charge at the onset of binding, corresponding to eq 1 , with $b=1.25$

In addition to $\sigma, \xi$, and $\kappa$, theory also points to the importance of the polymer chain stiffness, usually parameterized as the bare or intrinsic persistence length $l_{\mathrm{p}}{ }^{\mathrm{o}}$, and the expectation of diminished polyelectrolyte binding (larger $\sigma_{\mathrm{c}}$ ) for large $l_{\mathrm{p}}{ }^{\mathrm{o}}$ is also borne out by simulations. ${ }^{26,27}$ An additional polyelectrolyte property is charge mobility, and the ability of transient charges arising from proton migration to adapt to colloid charge patterns and so yield higher affinity has been suggested by experimen ${ }^{28}$ and simulations. In contrast to such "annealed" polyelectrolytes, strong or "quenched" polyelectrolytes can arrive at charge complementarity only through sequence distributions arising from synthesis conditions, as suggested by Feng et al. ${ }^{13}$

The purpose of the present work is to investigate the effects on colloid binding of these polyelectrolyte structural variables: chain stiffness, charge density, charge mobility, and sequence distribution. An attempt was made to focus on comparisons among or between anionic polyelectrolytes in which only one of these parameters varied. As examples of stiff polyelectrolytes, we employed hyaluronic acid (HA) and pectin. Charge mobility was provided by these two biopolyelectrolytes and poly (acrylic acid) (PAA). Charge density was investigated by using copolyelectrolytes made by copolymerization of acrylamide (AAm) and 2-acrylamido-2-methylpropanesulfonate (AMPS). Some degree of control of sequence distribution was sought by using copolymers prepared in $N, N$-dimethylformamide (DMF) and in water because reactivity ratios differ markedly in these two solvents. Our method is the construction and analysis of "phase boundaries", i.e., the ionic strength dependence of critical colloid charge over the range $0.010 \mathrm{M}<I<0.6 \mathrm{M}$. Our primary interest was protein binding, and we chose for this purpose bovine serum albumin (BSA), readily purified, inexpensive, and with known surface charge distributions. ${ }^{23,29}$ Because of the complexity of these distributions, companion studies were carried out with a spherical cationic-nonionic mixed micelle, dodecyltrimethylammonium bromide/Triton X-100 (DTAB/TX100 ), which provided as an additional advantage the ability to fix $\mathrm{pH}$ and hence the charge density of polycarboxylic acids. BSA-binding features not seen in the micelle-binding case could thus be more readily ascribed to aspects unique to the protein, i.e., charge and shape anisotropy. The results for micelle binding were consistent with characteristic simulations snapshots. For the protein case, visualizations of protein charge arrangements by protein electrostatic potential modeling were invoked to explain several features of interest.

\section{Experimental Section}

Materials. Heat shock, fatty acid-free bovine serum albumin (BSA) was purchased from Roche Diagnostics (Indianapolis, IN) (lot 93225920) and was used without further purification. Triton X-100 (TX-100) and dodecyltrimethylammonium bromide (DTAB) were purchased from Aldrich (St. Louis, MO).

Copolymers of poly(2-acrylamido-2-methylpropanesulfonate-coacrylamide) (AMPS-AAm) of varying AMPS content were synthesized
Table 1. Properties of Synthetic Polyelectrolytes

\begin{tabular}{lccrc}
\hline & $\begin{array}{c}\text { feed } \\
\text { composition }\end{array}$ & $\begin{array}{c}\text { actual } \\
\text { composition }\end{array}$ & \multicolumn{1}{c}{$M_{\mathrm{n}}$} & $\begin{array}{c}\text { conversion } \\
\%\end{array}$ \\
\hline H20-38 & $50: 50$ & $38: 62$ & 108000 & 10 \\
DMF50 & $50: 50$ & $52: 48$ & 45000 & 9.3 \\
PAMPS $^{a}$ & $100: 0$ & $100: 0$ & 100000 & \\
DMF20 $^{a}$ & $20: 80$ & $20: 80$ & $\sim 100000$ & 99 \\
DMF25 $^{a}$ & $25: 75$ & $25: 75$ & 200000 & 99 \\
\hline
\end{tabular}

${ }^{a}$ Reference 30 .

by free-radical polymerization in $N, N$-dimethylformamide (DMF) (MW 45000 estimated by SEC) and in water (MW approximately 100000 estimated by SEC), using monomer feed compositions of $0.50,0.25$, and 0.20 mole fraction AMPS. The AMPS homopolymer (PAMPS) was synthesized in water (MW on the order of 100000 ) and subsequently dialyzed and lyophilized prior to use. To avoid the use of subscripts in plots, the AMPS/AAm copolymers are referred to by their synthesis solvent and AMPS content, e.g., "DMF20". Poly(acrylic acid) (PAA) (Aldrich, MW 250 000) was neutralized with sodium hydroxide, dialyzed, and lyophilized. A second sample (Polysciences, MW 50000 ) was similarly prepared. In keeping with previous findings, ${ }^{16} \mathrm{MW}$ was seen to have no effect on the onset of soluble complexation. More detailed information on the polyelectrolytes synthesized is given in Table 1.

Pectin (CP Kelko, Lille Skensved, Denmark) "Genu test sample 0001-8D”, MW 86 000, 48\% esterified, was reported to have a blocky methyl ester distribution. Sodium hyaluronate (MW 900000 and 1200 000) was obtained from Shiseido (Yokohama, Japan). NaCl, 0.1 $\mathrm{N} \mathrm{HCl}$, and $0.1 \mathrm{~N} \mathrm{NaOH}$ were all certified ACS grade from Fisher Scientific.

Experimental Methods. Turbidimetric Titration. Turbidity measurements were made with a Brinkman PC 800 probe colorimeter (Westbury, NY) fitted with a $470-\mathrm{nm}$ filter and a $1.0-\mathrm{cm}$ path length optical probe. An automatic titrating system (ATS), designed and constructed in-house by K. W. Mattison and Y. Zhu, was used for programmed delivery of titrant from a $2.0-\mathrm{mL}$ microburet (Gilson). Transmittance was set to $100 \% \mathrm{~T}$ with Milli-Q water. For polyelectrolyte-protein titrations, a Corning $\mathrm{pH}$ meter, calibrated with $\mathrm{pH} 4.0$ and $7.0 \mathrm{pH}$ buffers, was used to measure solution $\mathrm{pH}$ upon each addition of $\mathrm{HCl}$.

BSA and polymer solutions were prepared at $2.5 \mathrm{~g} / \mathrm{L}$ and $0.5 \mathrm{~g} / \mathrm{L}$, respectively, in $\mathrm{NaCl}$ solution of the desired ionic strength. BSA solutions were adjusted to $\mathrm{pH} 9.0$ to prevent self-aggregation and subsequently stirred for $1 \mathrm{~h}$ prior to filtering. Polymer solutions were allowed to stir at least $2 \mathrm{~h}$; pectin samples, overnight. All solutions were prepared with Milli-Q water and filtered through Sartorius 0.22$\mu \mathrm{m}$ membrane filters prior to use. Protein and polymer samples prepared as described above were combined $1: 1(\mathrm{v} / \mathrm{v})$ for final concentrations of $1.25 \mathrm{~g} / \mathrm{L}$ and $0.25 \mathrm{~g} / \mathrm{L}$, respectively. Pectin samples were adjusted to neutral $\mathrm{pH}$ just prior to mixing with protein solutions. All solutions were titrated with $0.1 \mathrm{~N} \mathrm{HCl}$ from $\mathrm{pH} 8$ to a $\mathrm{pH}$ at which solutions were visibly turbid. Following titration, plots of $100 \% \mathrm{~T}$ vs $\mathrm{pH}$ were constructed to determine $\mathrm{pH}_{\mathrm{c}} \cdot{ }^{23-25}$ The values of $\mathrm{pH}_{\mathrm{c}}$ could be obtained with a precision of \pm 0.1 , as found from duplicate measurements.

DTAB, TX-100, and polymer solutions, with the exception of pectin, were prepared at $500 \mathrm{mM}, 40 \mathrm{mM}$, and $1.0 \mathrm{~g} / \mathrm{L}$ concentrations, respectively, in $\mathrm{NaCl}$ solution of the desired ionic strength. Polyelectrolyte-micelle titrations were done with the same polymers used in the protein study except for DMF25, which was replaced by DMF20 due to the low availability of the former. For pectin titrations, the concentrations of each solution were halved. PAA and pectin samples were each adjusted during preparation to a $\mathrm{pH}$ corresponding to a degree of ionization $\alpha=0.50$. Titration solutions were prepared by mixing polymer and TX-100 1:1 (v/v) to give $0.5 \mathrm{~g} / \mathrm{L}$ and $20 \mathrm{mM}$ final concentrations, respectively (for pectin titrations, final concentrations were $0.25 \mathrm{~g} / \mathrm{L}$ and $10 \mathrm{mM}$, respectively). DTAB (500 mM or $250 \mathrm{mM}$ 
in the case of pectin) was then added from a microburet until solutions were visibly turbid. $Y_{\mathrm{c}}$, the critical DTAB mole fraction at the onset of binding, measurable with a precision of \pm 0.02 , was plotted vs ionic strength to construct micelle- polyelectrolyte phase boundaries. ${ }^{12,13,15,16}$

Dynamic Light Scattering. DLS measurements were carried out at a scattering angle of $90^{\circ}$ and at $25 \pm 1{ }^{\circ} \mathrm{C}$ with a Brookhaven system (Holtsville, NY) equipped with a 256-channel digital autocorrelator (BI9000 AT) employing a 488-nm $100 \mathrm{~mW}$ Ar laser. A 400- $\mu \mathrm{m}$ pinhole aperture was employed for the EMI photomultiplier tube, and decalin (dehydronaphthalene) was used as the refractive index matching fluid to reduce stray light. Because free protein and protein-polymer complex could coexist, we analyzed the autocorrelation functions by using both CONTIN and NNLS at $\mathrm{pH}<\mathrm{pH}_{\mathrm{c}}$. We used CONTIN alone only at $\mathrm{pH}>\mathrm{pH}_{\mathrm{c}}$ because, under these conditions, the protein is the only significant scatterer. The application of these methods to such systems has been reported in detail. ${ }^{31-33}$

Simulations. Computer simulations were used with the goal of interpreting experimental data and clarifying the importance of some of the observed effects. In this context, Monte Carlo simulations were performed according to the Metropolis algorithm in the grand canonical ensemble. A coarse grain model is used to generate off-lattice threedimensional polyelectrolyte chains containing a variable number of jointed, solvent-excluded, hard spheres $N$. A sphere is considered to be a physical monomer of radius $R_{\mathrm{m}}=l_{\mathrm{B}} / 2=3.57 \AA$, where $l_{\mathrm{B}}$ represents the Bjerrum length at $298 \mathrm{~K}$. Each monomer can carry a negative charge on its center or can be neutral. The charges on the polyanion are considered here as in equilibrium with a bulk of fixed chemical potential. Water is treated as a dielectric medium with a dielectric constant $\epsilon_{\mathrm{r}}=78.5$. The micelle or globular protein is represented as an impenetrable, solvent-excluded, uniformly charged sphere. The radius of the sphere is noted $R_{\mathrm{p}}$, and its positive charge is assumed to be concentrated into a point located on its center. It is assumed that both the polyelectrolyte and micelle or globular protein have the same dielectric constant as the solvent. The long-range repulsive electrostatic potential along the distance $r_{i j}$ between charged units $i$ and $j$ is described via a screened Debye-Hückel potential: ${ }^{34}$

$$
u_{\mathrm{el}}\left(r_{i j}\right)=\frac{z_{i} z_{j} e^{2}}{4 \pi \epsilon_{\mathrm{r}} \epsilon_{0} r_{i j}} \frac{\exp \left[-\kappa\left(r_{i j}-\left(R_{i}+R_{j}\right)\right)\right]}{\left(1+\kappa R_{i}\right)\left(1+\kappa R_{j}\right)}
$$

the overall effect of free ions on monomer-monomer and monomermicelle interactions are described via the dependence of the inverse Debye screening length $\kappa$ on the electrolyte concentration. The stiffness of the chain is controlled by freely rotating connections between rigid segments connecting the monomers. The intrinsic chain stiffness is adjusted by a square potential with variable amplitude to vary its strength, and the bending energy is given by

$$
E_{\mathrm{tor}}=\sum_{i=2}^{N} k_{\mathrm{ang}}\left(\theta_{i}-\theta_{0}\right)^{2}
$$

where $\theta_{i}$ represents the angle formed by the vectors consecutive monomers $\mathbf{r}_{i-1}-\mathbf{r}_{i}$ and $\mathbf{r}_{i+1}-\mathbf{r}_{i}$ and $\theta_{0}=180^{\circ} . k_{\text {ang }}$ defines the chain stiffness and is here expressed in $k_{\mathrm{B}} T / \mathrm{deg}^{2}$ units.

To sample low energy conformations, the monomer positions are randomly modified by specific movements such as pivot, end-bond, and kink-jump. After each elementary random move, the change in energy, $\Delta E$, is considered and the Metropolis ${ }^{35}$ selection criterion is employed to either select or reject the movement. The polyelectrolyte is considered as adsorbed when at least one monomer is in contact with the micelle, i.e., the distance between their centers is $<R_{\mathrm{p}}+2 R_{\mathrm{m}}$, during more than $50 \%$ of the equilibration period.

Because of their connectivity along the polyelectrolyte chain, charged monomers strongly interact and their acid/base properties are different from ideal systems. The difference in the acid/base properties between the isolated monomer and the monomer within the polyelectrolyte is expressed by

$$
\Delta \mathrm{p} K=\mathrm{p} K-\mathrm{p} K_{\mathrm{a}}=\mathrm{pH}-\mathrm{p} K_{\mathrm{a}}-\log \frac{\alpha}{1-\alpha}
$$

where $\mathrm{p} K_{\mathrm{a}}$ represents the dissociation constant of a monomer in the absence of electrostatic interactions and $\alpha$ the degree of ionization. $\Delta \mathrm{p} K$ largely depends on polyelectrolyte length, complex formation, and polyelectrolyte degree of ionization, as well as on the ionic concentration and presence of an oppositely charged sphere. In this model, after a given number of MC steps to equilibrate the polyelectrolyte conformation, a monomer is chosen at random, and depending on the solution $\mathrm{pH}$, its charge state is switched on or off, respectively. The energy change, $\Delta E$, that determines the probability for accepting (according to the Metropolis Monte Carlo criteria) the new charge state is then the sum of the change in electrostatic interaction $\Delta E_{c}$ and a term that corresponds to the change in free energy, ${ }^{36,37}$

$$
\Delta E=\Delta E_{c} \pm k_{\mathrm{B}} T\left(\mathrm{pH}-\mathrm{p} K_{\mathrm{a}}\right) \operatorname{In}(10)
$$

When a monomer is protonated, the plus sign is used, whereas when the monomer is deprotonated, the minus sign is required. To consider $\mathrm{pH}$ variations in the solution, the value of $\mathrm{pH}-\mathrm{p} K_{\mathrm{a}}$ in eq 5 is then adjusted for each simulation run (input parameter). For each simulation run at a given $\mathrm{pH}-\mathrm{p} K_{\mathrm{a}}$ value, after energy minimization, the polyelectrolyte degree of ionization $\alpha$ is directly measured by considering the number of charged monomers from the analysis of the equilibrated conformations.

Protein Electrostatic Potential Modeling. Molecular protein modeling was carried out by DelPhi version 98.0 (Molecular Simulations, San Diego, CA). The crystal structure of dimeric human serum albumin (HSA) was obtained from the Research Collaboratory for Structural Bioinformatics (RCSB) Protein Data Bank (PDB ID 1AO6) (www.rcsb.org/pdb) and edited to more closely resemble monomeric bovine serum albumin (BSA) before calculating the electrostatic potential. Chain B of the dimer, identical to chain A, was deleted. The charges of amino acids on proteins were determined by following the simple charge model of Tanford. ${ }^{38}$ Partial charges were assigned to each amino acid capable of bearing a net charge at any given $\mathrm{pH}$. These assignments were based on intrinsic $\mathrm{p} K$ values determined from published titration curves. ${ }^{23}$ Three $\mathrm{N}$-terminal amino acids were missing from the PDB file; this was corrected by assigning to the terminal amino acid a net charge corresponding to the sum of its own charge and the charges of the three deleted amino acids. The edited structure was placed in the center of a grid box to provide maximum working surface. The resolution was set to 101 grid points per axis. The dielectric constants of the protein and solvent were set to 2.5 and 80 , respectively. The electrostatic potential around the protein was calculated as a function of $\mathrm{pH}$ and ionic strength by nonlinear solution of the PoissonBoltzmann equation, converging after 500 iterations. Red and blue potential surfaces around the proteins were set to represent $-0.1 \mathrm{kT} / \mathrm{e}$ and $0.1 \mathrm{kT} / \mathrm{e}$, respectively.

\section{Results and Discussion}

Critical Conditions for Complex Formation. Equation 1 describes the condition for the appearance of a bound state, at a fixed polyelectrolyte linear charge density, in terms of the relationship between the critical surface charge density $\sigma_{\mathrm{c}}$ and the Debye-Hückel screening parameter $\kappa$. To express this in experimental terms, we first recognize that, for 1:1 electrolytes in water at ambient temperature, $\kappa\left(\mathrm{nm}^{-1}\right)=3.281 * \sqrt{ } I$, and express eq 1 as

$$
\sigma_{\mathrm{c}} \xi \sim I^{a}
$$

or, at a fixed polyelectrolyte charge density,

$$
\sigma_{\mathrm{c}}=K I^{a}
$$




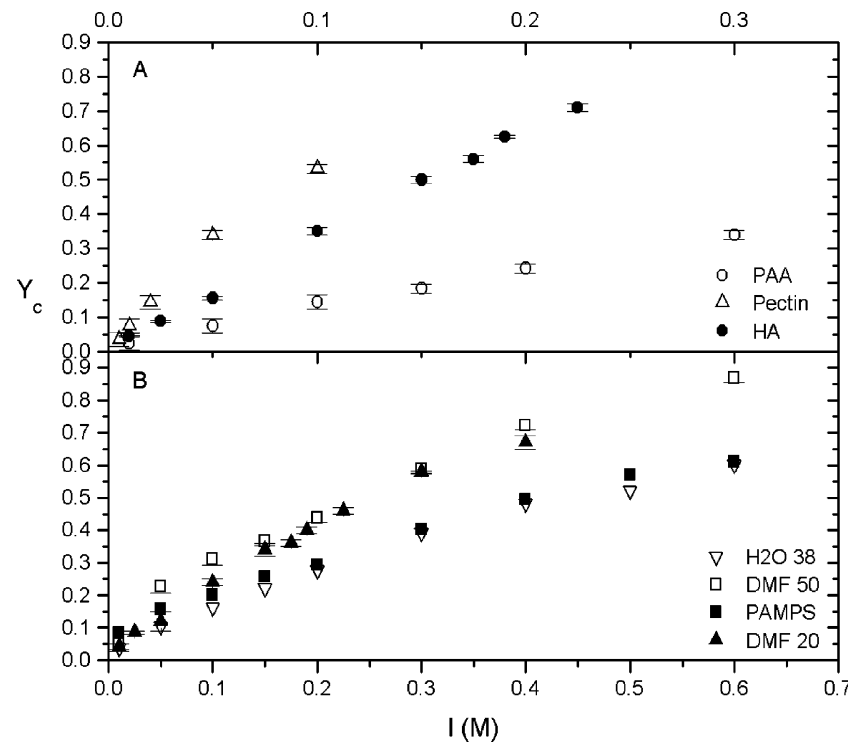

Figure 1. Critical conditions for complex formation between polyanions and DTAB/TX-100 mixed micelles, expressed as the ionic strength dependence of $Y_{\mathrm{C}}$, the micellar mole fraction of cationic surfactant at conditions of incipient complexation. (A) weak polyelectrolytes, (B) strong polyelectrolytes.

where the exponent $a$ is related to that in eq 1 by $a=b / 2$. Equations 6 describe the ionic strength dependence of the point of incipient complex formation induced by a continuous increase in colloid charge. For proteins, this increase arises from a change in $\mathrm{pH}$, as is also the case for micelles with titratable headgroups or dendrimers with titratable termini. Extraction of $\sigma$ from experimental data is possible in the last two cases by using potentiometric titration to obtain surface potentials and converting those to surface charge densities via some form of the Poisson-Boltzmann equation. For mixed micelles, a continuous increase in colloid surface charge density corresponds to a change in $Y$, the mole fraction of the ionic surfactant. When a potentiometric probe is included in the mixed micelle, $\sigma$ can be measured directly, but more commonly it is presumed to be linear with $Y$ or, more precariously, with net protein charge, $Z$. Thus, experiments that adhere to eqs $6 \mathrm{a}$ and $\mathrm{b}$ support theory, especially if they make it possible to test the identification of the prefactor $K$ or exponent $a$ with physical parameters such as polyelectrolyte chain stiffness (embodied in the bare persistence length, $\left.l_{\mathrm{p}}{ }^{\circ}\right)$, or polyelectrolyte charge density $\tau$, where $\tau$ is a normalized charge density equal to one when every repeat unit of a vinylic polymer bears a full charge. The theory that leads to eq 1 is a single-molecule theory that does not consider multichain or colloid-colloid interactions; the absence of any influence of polymer concentration or micelle concentration on $Y_{\mathrm{c}}$ demonstrates that eqs $6 \mathrm{a}$ and $\mathrm{b}$ are appropriate for the current experimental results. ${ }^{16}$ Regardless of theory, linearization of the $I$ dependence of $Y_{\mathrm{c}}$ or $Z_{\mathrm{c}}$ facilitates comparisons among different polyelectrolytes or between different ionic strength regimes for a single polyelectrolyte. Examination of plots of $\log Y_{\mathrm{c}}$ or $\log$ $Z_{\mathrm{c}}$ vs $\log I$ over one or 2 orders of magnitude in $I$ makes it possible to identify a linear regime and extract the value of $a$. The subsequent plots of $Y_{\mathrm{c}}$ or $Z_{\mathrm{c}}$ vs $I^{a}$ provide a more stringent test of the scaling term and allow us to decide whether $a$ itself is dependent on polyelectrolyte. The procedures for expressing the results as $K I^{a}=Y_{\mathrm{c}}$ or $Z_{\mathrm{c}}$ will be explained separately for micelles and proteins.

Parts A and B of Figure 1 show the ionic strength dependence of $Y_{\mathrm{c}}$ for all polyelectrolytes. Linearization of micelle binding data was done by first subtracting from $Y_{\mathrm{c}}$ its extrapolated value

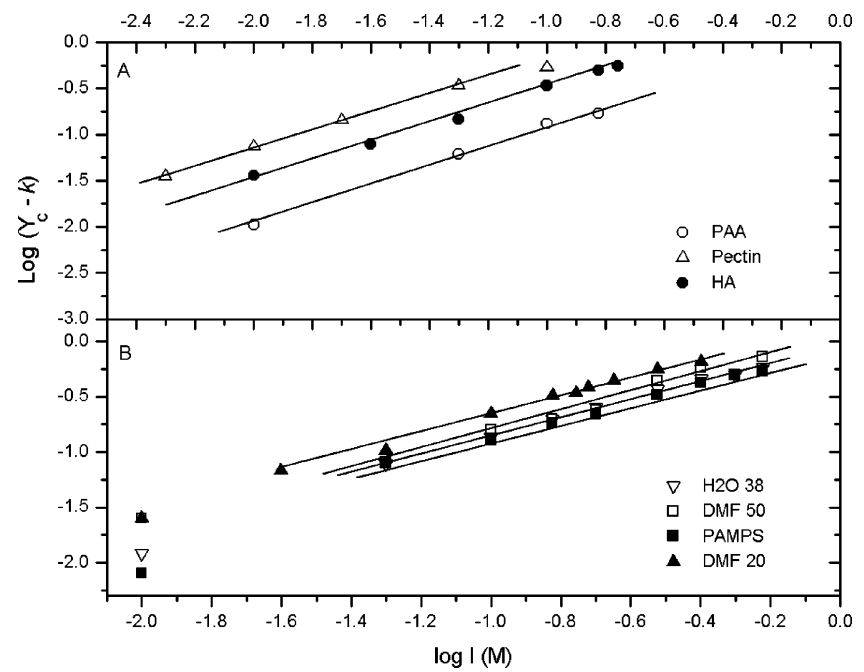

Figure 2. Log-log plots based on data from Figure 1. (A) weak polyelectrolytes, (B) strong polyelectrolytes. $Y_{\mathrm{c}}$ values are adjusted by subtracting from $Y_{\mathrm{c}}$ its extrapolated value at $I=0$ (referred to as $k$ in subsequent log-log plots); this constraint is removed in later sections. The linear region used to determine the exponent $a$ is shown.

at $I=0$, " $k$ " for Figure $1 \mathrm{~A}$ and B. This constraint that forces adherence to eq $6 \mathrm{~b}$ is later removed. The subsequent $\log -\log$ plots (Figure 2A and B) gave a value of $a=0.95( \pm 0.04)$ for all polycarboxylic acids, while for all AMPS-containing polymers, $a=0.85( \pm 0.03)$. Because of the adjustment forcing $Y_{\mathrm{c}}$ to 0 at $I=0$, the vertical shift of the parallel curves in Figure $2 \mathrm{~A}$ or $\mathrm{B}$ should not be interpreted. The adjustment for Figure $2 \mathrm{~A}$, however, is almost negligible, so that the vertical displacement corresponds to differences in the prefactor $K$, attributable here to increasing $l_{\mathrm{p}}{ }^{\mathrm{o}}$ (from bottom to top).

Close inspection of the nonlinearized $Y_{\mathrm{c}}$ vs $I$ curves presented in Figure 1B might imply a plateau at high $Y$ values, possibly suggesting counterion condensation. Previous studies ${ }^{39,40}$ on surface potential and mobility of ionic/nonionic surfactants did in fact reveal a plateau in mobility at high ionic surfactant composition, which was attributed to micelle counterion condensation. The plateau onset at $Y>0.8$ was, however, above nearly all the $Y_{\mathrm{c}}$ values in this study.

Figure 3 expresses conditions for the onset of polyelectrolyte-protein binding as the ionic strength dependence of the net protein charge at the critical $\mathrm{pH} . Z_{\mathrm{c}}$ values are found both above and below 0 . To avoid logarithms of negative numbers, we plotted $\log \left(Z_{\mathrm{c}}+10\right)$ vs $\log I$ (Figure $4 \mathrm{~A}$ and $\mathrm{B}$ ) and observed linearity at $I<0.36 \mathrm{M}$ for the AMPS-containing polymers and over smaller ranges of $I$ for HA or pectin. For all polyelectrolytes except for the low conversion copolymer synthesized in water, we obtained $a=0.45( \pm 0.05)$ (Figure 4B). Figure 4 reveals an apparent contradiction: the phase boundaries pass through $Z$ $=0\left(\log \left(Z_{\mathrm{c}}+10\right)=1.0\right)$, indicating that binding involves a local protein domain ("positive patch") with no special effect at $\mathrm{pH}=\mathrm{pI}$; nevertheless, the variation of the global protein charge $Z_{\mathrm{c}}$ with $I^{a}, a=1 / 2$ is compellingly simple. This relationship between the global protein charge and a local effective patch charge will be discussed later.

Effect of Charge Mobility. While charges in AMPScontaining polymers are spatially fixed ("quenched"), charges associated with weak polyacids or polybases with degrees of ionization $\alpha$ less than unity are subject to equilibrium between protonated and deprotonated states and may thus be considered mobile ("annealed"). There are three such polyanions in this study: PAA, HA, and pectin. Their degree of ionization $\alpha$ can 


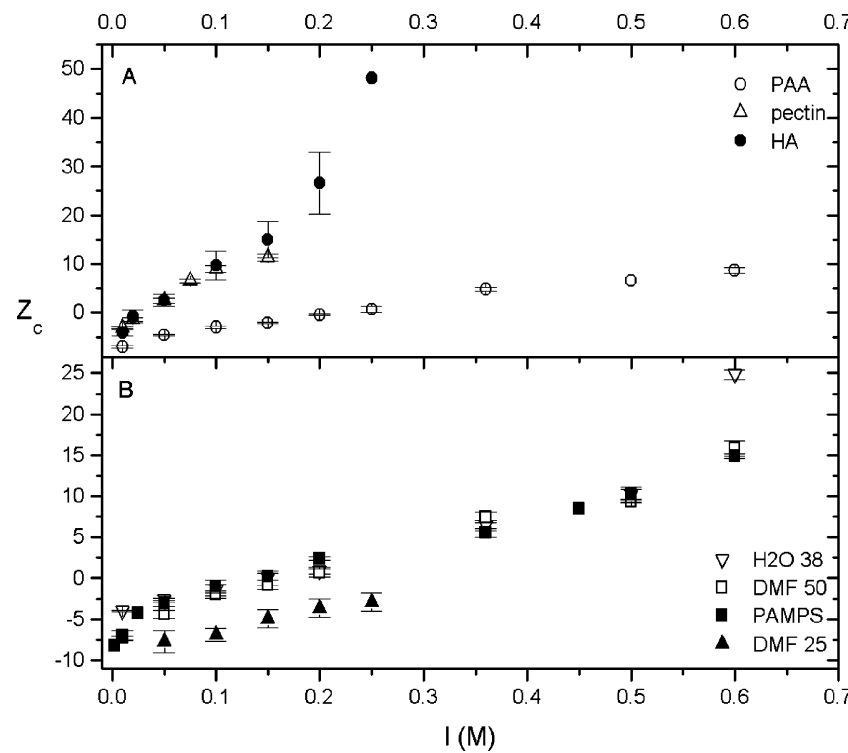

Figure 3. Critical conditions for complex formation between polyanions of this study and BSA, expressed as the ionic strength dependence of the net protein charge at conditions of incipient complexation. (A) weak polyelectrolytes, (B) strong polyelectrolytes.

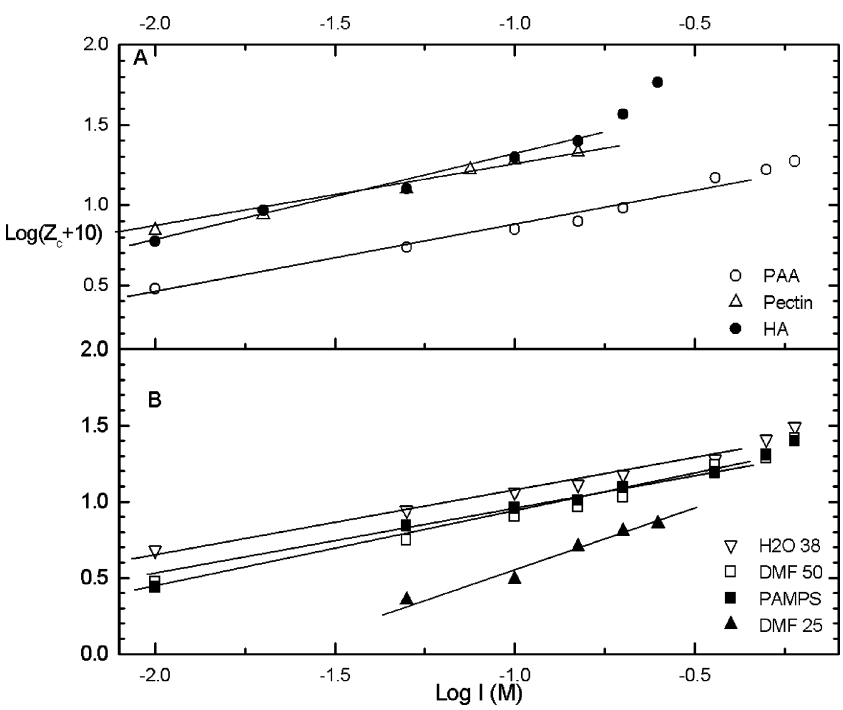

Figure 4. Log-log plot using data from Figure 3. (A) weak polyelectrolytes, (B) strong polyelectrolytes. Net protein charge values are adjusted by +10 in order to avoid negative arguments of the log. Lines are drawn to indicate the regions used to determine the exponent $a$.

be fixed by buffer selection for micelle-binding experiments, which will be presented first. For PAA, $\alpha=0.5$ was chosen to facilitate comparison with the DMF50 copolymer; this degree of ionization is attained at a $\mathrm{pH}$ between 4.6 and 6.5, depending on the ionic strength of the solution, ${ }^{41}$ here varying from 0.010 to $0.60 \mathrm{M}$. As will be explained below, the $\mathrm{p} K_{\mathrm{a}}$ 's of pectin and HA were low enough to ensure nearly full ionization. As seen in Figure 5A, PAA showed the strongest micelle affinity. This strong binding relative to the quenched polyelectrolytes is due to charge mobility: the ability of protons to migrate in order to form a sequence more favorable to binding than the statistically average sequence. This effect is illustrated by simulation snapshots of a weak annealed polyelectrolyte binding to an oppositely charged sphere in Figure $6 \mathrm{~A}-\mathrm{F}$, in which charges migrate to the binding region and are depleted from locally unbound repeat units, i.e., $\tau_{\text {eff }}>\tau_{\text {av }}$. Here, the polyanion degree of ionization is controlled by adjusting the $\mathrm{pH}-\mathrm{p} K_{\mathrm{a}}$ value of

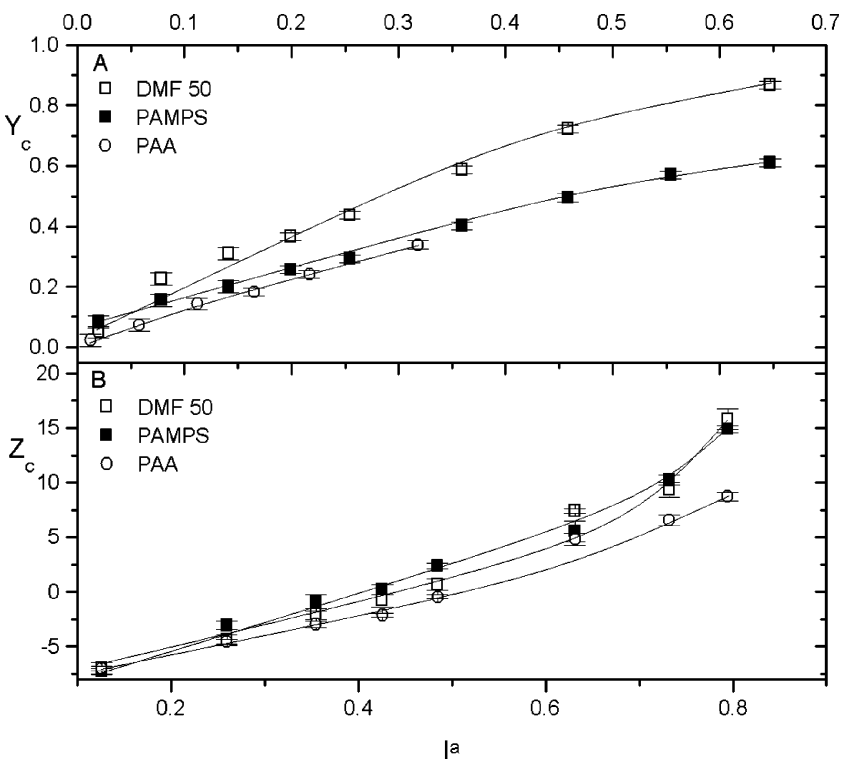

Figure 5. Phase boundaries for polyelectrolyte-micelle $(A)$ and polyelectrolyte-protein systems (B), comparing weak and strong polyelectrolytes of similar charge density. Values of $a$ are 0.85 and 0.45 in $A$ and $B$, respectively, for all polymers, except for $a=0.95$ for PAA in A. The curved lines are to guide the eye.

the solution. For the annealed polyanion, in response to the decrease of $\alpha$ with $\mathrm{pH}-\mathrm{p} K_{\mathrm{a}}$, charges migrate to the binding region and are depleted from locally unbound repeat units. Consequently, binding is more readily achieved by the annealed polyanion (see $\mathrm{F}$ and $\mathrm{L}$ conformations). For the quenched polyanion, charges are regularly distributed along the polymer backbone. In this case, charge sequence distribution plays an important role in the final complex formation by stabilizing loop formation (optimal charge arrangement) for $\alpha=0.32,0.18$, and also presumably at intermediate values of $\alpha$.

An additional consideration in the case of micelles and PAA is the possibility of hydrogen bonding between the carboxylic acid groups in PAA and ethylene oxide groups in Triton X-100. As shown by Yoshida et al., ${ }^{28}$ such $\mathrm{H}$ bonding is significant at $\alpha<0.2$, and negligible at $\alpha>0.8$, and therefore could make a contribution. However, such a nonelectrostatic contribution to micelle binding should uniformly depress the measured values of $Y_{\mathrm{c}}$ at low $\alpha$, thus leading to a negative value of $Y_{\mathrm{c}}$ in the limit of $I=0$, which is not observed in Figure 5A.

$\mathrm{pH}$ is not an independent variable in protein binding studies (Figure $5 \mathrm{~B}$ ) because the critical $\mathrm{pH}$ is determined by proteinpolyelectrolyte affinity. In the most problematic case of PAA, $\mathrm{pH}_{\mathrm{c}}$ varies from 6.8 at $I=0.01 \mathrm{M}$ to 4.9 at $I=0.6 \mathrm{M}$, and consequently, the degree of ionization $\alpha$ of this weak polyacid can vary from 0.5 to 0.75 in the limits of high and low $I$. However, if $\left(\mathrm{pH}-\mathrm{p} K_{\mathrm{a}}\right) \geq 2$, we can assume complete ionization, i.e., no charge mobility. This is nearly the case for pectin, for which literature values of $\mathrm{p} K_{\mathrm{a}}$ vary from 4.0 to 4.4 in pure water, and from 3.5 to 4.0 at $I=0.05 \mathrm{M}$. For pectin, $\mathrm{pH}_{\mathrm{c}}$ values ranged from 5.9 to 4.4 , such that the condition $(\mathrm{pH}$ $\left.-\mathrm{p} K_{\mathrm{a}}\right) \geq 2$ is satisfied at $I \leq 0.1 \mathrm{M}^{4}{ }^{42}$ Conflicting values are found for the $\mathrm{p} K_{\mathrm{a}}$ of HA: Cleland ${ }^{43}$ reported $2.8<\mathrm{p} K_{\mathrm{a}}<3.2$, depending on $I$ and $\alpha$; a more recent report gives $\mathrm{p} K_{\mathrm{a}}$, in $I=$ $0.05 \mathrm{M}$, as $3.5 \pm 0.1$ independent of $\alpha,{ }^{44}$ but these values are also low enough to assume full ionization for HA over most of the $\mathrm{pH}_{\mathrm{c}}$ range. Therefore, the condition $\left(\mathrm{pH}-\mathrm{p} K_{\mathrm{a}}\right) \geq 2$ was satisfied at $I \leq 0.15 \mathrm{M}$. On the other hand, the larger values of $\mathrm{p} K_{\mathrm{a}}$ for PAA result in $\alpha$ at $\mathrm{pH}_{\mathrm{c}}$ ranging from 0.75 to 0.50 as $I$ increases from 0.01 to $0.6 \mathrm{M}$. Despite this complication of determining the degree of charge for PAA at different condi- 


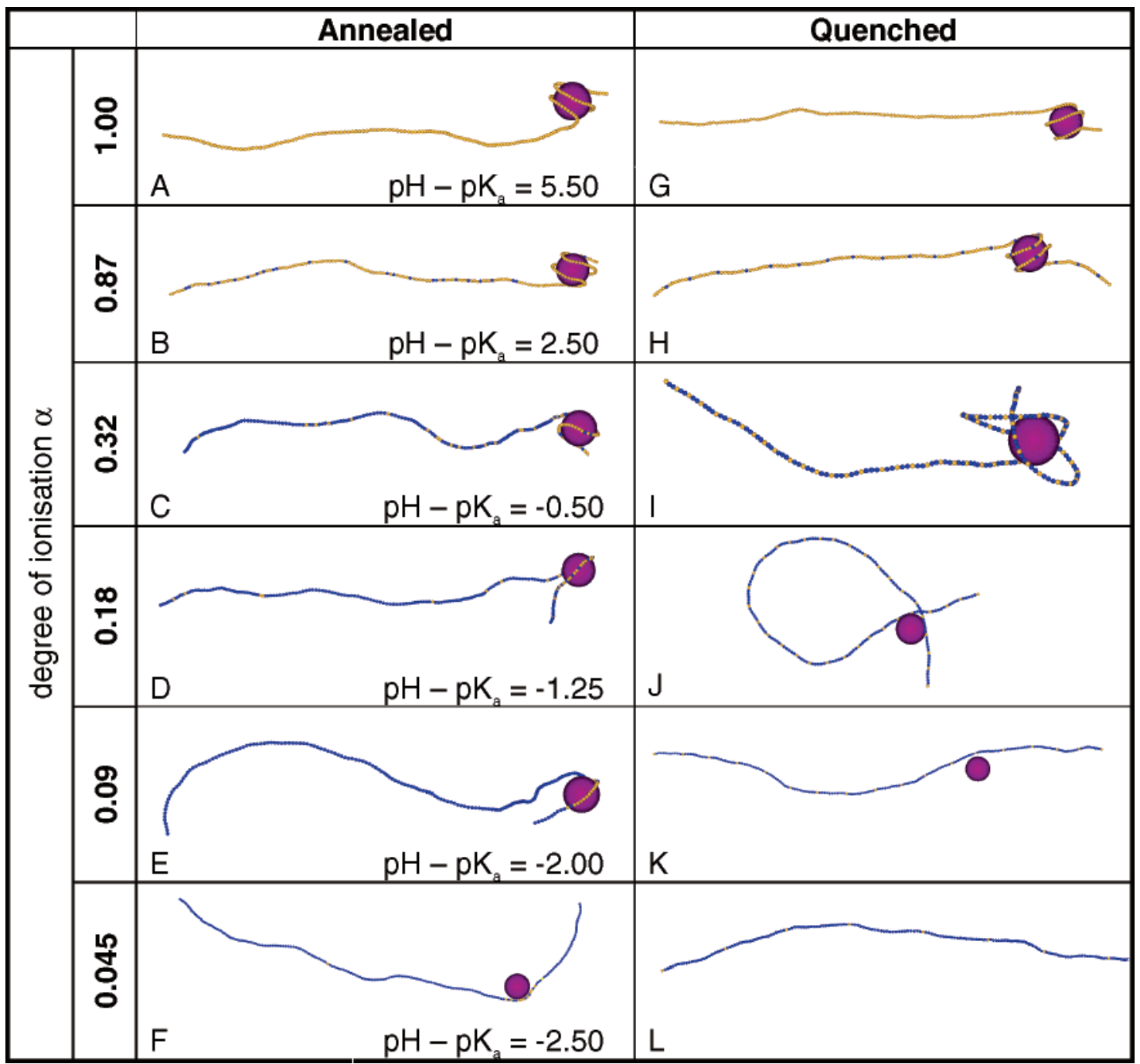

Figure 6. Equilibrated conformations of a weak polyanion, forming a complex with an oppositely charged sphere. $I=0.001 \mathrm{M}, R_{\mathrm{p}}=35.7 \AA$ $=+75 \mathrm{mC} / \mathrm{m}^{2}, N=200$ and $k_{\text {ang }}=0.02 \mathrm{kB} T / \mathrm{deg}^{2}$.

tions, we focus in this section on PAA because resolving effects of charge from chain stiffness for HA and pectin is even more problematic. The range of $\alpha$ for PAA places its charge density intermediate between those of PAMPS and DMF50, so its stronger binding relative to both these quenched polyelectrolytes, as seen in Figure 5B, must arise from charge mobility. We determined that $\mathrm{pH}_{\mathrm{c}}$ for $\mathrm{BSA}-\mathrm{PAA}$ at $I=0.6 \mathrm{M}$ was such that $\alpha=0.5$, so at this ionic strength, direct comparison between PAA and DMF50 can be made. This comparison establishes stronger binding for the annealed copolymer at fixed $\tau=0.5$.

Effect of Chain Stiffness. The effect of polyelectrolyte chain stiffness on colloid binding affinities may be evaluated by comparing the highly flexible PAA $\left(l_{\mathrm{p}}{ }^{\mathrm{o}}=1.2 \pm 0.5 \mathrm{~nm}\right)^{45}$ to pectin and hyaluronic acid, $\mathrm{HA}\left(l_{\mathrm{p}}{ }^{\mathrm{O}}=7 \pm 2 \mathrm{~nm},{ }^{46,47}\right.$ and $4.1 \pm$ $1 \mathrm{~nm},{ }^{48}$ respectively) (See Table 2 ). Because the charge density $(\tau)$ of PAA here is at least twice that of pectin and HA, we include AMPS-containing copolymers ("DMF20" or "DMF25") of the same (relatively low) linear charge density as pectin and HA, i.e., average spacing between charges about $12 \AA .49$

The intuitive expectation that binding is diminished by polymer chain stiffness, supported by theory ${ }^{26}$ and simulations, ${ }^{27,53,54}$ is borne out by the results for micelle binding in Figure 7A. The prefactor in eq $6 \mathrm{~b}$, now seen as the slope of the $Y_{\mathrm{c}}$ vs $I^{a}$ plots (s), increases nearly proportionally to $l_{\mathrm{p}}{ }^{\mathrm{o}}$ (See Figure 7, inset), although the strong binding and low slope for PAA could be due to either its higher charge density or to charge
Table 2. Literature Values of Polyelectrolyte Persistence Lengths ${ }^{a}$

\begin{tabular}{lcl}
\multicolumn{1}{c}{ polyelectrolyte } & $l_{\mathrm{p}}{ }^{\circ}(\mathrm{nm})$ & \multicolumn{1}{c}{$\mathrm{t}$} \\
\hline PAA $^{b}$ & 1.2 & $>0.5$ \\
AMPS-AAm copolymers $^{c}$ & $1-2$ & variable \\
PAMPS $^{d}$ & 2.4 & 1.0 \\
pectin $^{e}$ & 7.0 & 0.25 \\
HA $^{f}$ & 4.1 & 0.25 \\
\hline
\end{tabular}

a Unless otherwise noted, these are the bare (intrinsic) persistence lengths, i.e., in the limit of infinite ionic strength. ${ }^{b}$ At $I=1.5 \mathrm{M} .{ }^{45}{ }^{c}$ Based on $l_{p}{ }^{\circ}$ values for AAm and AMPS homopolymers. ${ }^{50,51}{ }^{d}$ From ref 52 ${ }^{e}$ Based on extrapolation of $l_{\mathrm{p}}$ vs $\left(\kappa^{-1 / d}\right)$ to $\left(\kappa^{-1 / d}\right)=0$, where $d$ is equal to the polyelectrolyte charge spacing. ${ }^{46,47}$ f Obtained at $I=0.5 \mathrm{M}\left(d / \kappa^{-1}\right.$ $>1) \cdot{ }^{48}$

mobility discussed in the previous section. Theory ${ }^{26}$ indicates that polyelectrolyte adsorption onto spheres is promoted by a reduction in $l_{1}$, a chain stiffness parameter that is an expansion factor for the end-to-end chain length in terms of bare Kuhn length. In agreement with this theory, increased binding strength is seen with a decrease in chain stiffness for three polyelectrolytes with similar charge densities: DMF20/25, HA, and pectin.

Binding to BSA (Figure 7B) does not entirely follow the expected effect of chain stiffness. While the protein affinities of the two stiff polymers are clearly diminished with respect to the flexible ones, pectin and HA show nearly equal values of 


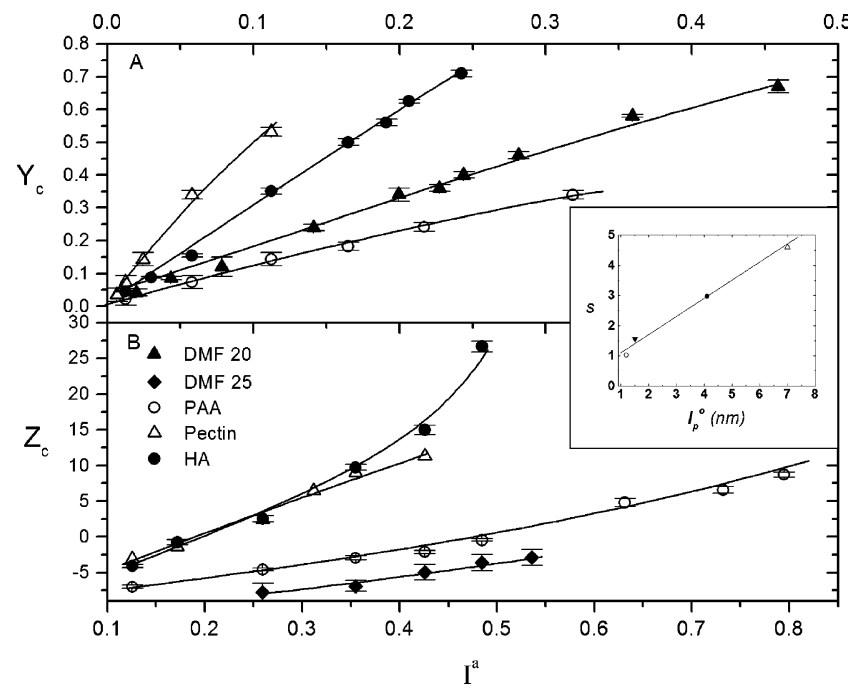

Figure 7. Phase boundaries for weak polyelectrolytes binding to cationic mixed micelles (A) and BSA (B). DMF20 and DMF25 are strong polyelectrolytes with charge densities comparable to the rigid weak polyelectrolytes pectin and HA. Values of $a$ as given for Figure 5. Inset: dependence of the slope of the lines in A, $s$, on $I_{p}^{\circ}$, symbols as in Figure 7A with $(\boldsymbol{\nabla})$ representing all AMPS-containing copolymers.

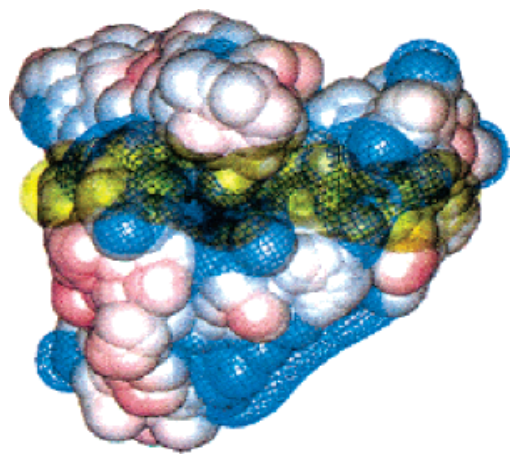

Figure 8. Human serum albumin (HSA) with hyaluronic acid (HA) (shown in green) superimposed on its binding site. ${ }^{23}$

$Z_{\mathrm{c}}$. BSA in the vicinity of $Z=0$ exhibits a relatively flat positive domain (see Figure 8) that accommodates a 5-nm length of the locally stiff polyanion HA; ${ }^{23}$ further increase in chain stiffness does not impede binding to this protein domain. For small spherical micelles, the effect of polymer stiffness is obviously more important. With regard to the two flexible polyelectrolytes, the stronger protein binding of DMF25 vs PAA was unexpected because PAA should have both more chain flexibility and more charge mobility than the copolymer. Repulsive forces between the polyanion and the negative domain of the protein must be considered, particularly at $Z<0$, corresponding to $\mathrm{pH}>5$. Here, PAA is largely ionized, attracted to the positive domain of BSA but repelled by negative protein regions, and upward $\mathrm{p} K$ shifts of PAA are not sufficient to suppress its local ionization. It may be easier to find in DMF25 a region rich in anionic residues, bracketed by essentially uncharged runs of AAm, an arrangement that minimizes repulsion while retaining attraction, thus yielding for DMF25 a higher protein affinity than that of PAA. These statistical sequences of DMF25 may also be a factor in its stronger binding than HA and pectin, all three polymers having similar average charge densities. The biosyntheses of HA and pectin are likely to yield isolated charges, with HA and $50 \%$ esterified pectin containing one ionizable group per disaccharide.

Effect of Sequence Distribution. In this section, we consider low-persistence length and strong polyelectrolytes of variable

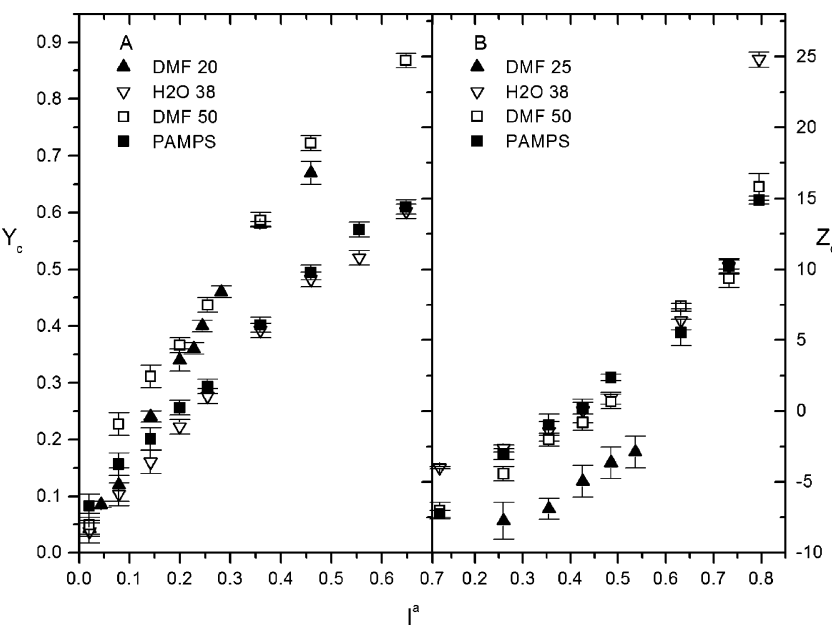

Figure 9. Critical conditions for complex formation between strong, flexible chain polyelectrolytes and (A) cationic micelles or (B) BSA. The value of a for all polyelectrolytes binding to DTAB/TX-100 is 0.85 ; for protein binding, $a$ is 0.45 .

charge density and variable sequence distribution. Independent control of the latter property is not easily accomplished, but some control is possible through variation of reactivity ratios. In the present case, $r_{1}=1.00$ and $r_{2}=1.02$ for polymerization in $\mathrm{DMF}^{55}$ and $r_{1}=0.49$ and $r_{2}=0.98$ for polymerization in water, ${ }^{56}$ where monomer 1 is AMPS and monomer 2 is acrylamide. Thus, for a copolymer prepared in water, AMPS residues tend to occur as isolated units, whereas both residues follow random arrangement in copolymers made in DMF. A consequence of the small value of $r_{1}$ for polymerization in water is composition drift, yielding highly heterogeneous (albeit interesting) polymers at high conversion, so we focus on lowconversion material. This sample, labeled $\mathrm{H} 2 \mathrm{O} 38$, is the product of aqueous copolymerization from an equimolar monomer feed ratio at $10 \%$ conversion and was found to contain $38 \mathrm{~mol} \%$ AMPS

Because there are few similarities between the relative affinities of the different polyelectrolytes in the case of micelles vis-à-vis proteins, we consider the two colloids separately. Equation 1 (derived decades ago in the absence of any consensus on the length or presence of the loops) indicates that the dependence of $\sigma_{\mathrm{c}}$ on $I^{a}$ (i.e., the slopes of Figure 9A) should vary inversely with $\tau$, and this is observed for the micelle binding of three polymers synthesized in DMF (DMF20, DMF50, and PAMPS). On the other hand, two features of Figure 9A are inconsistent with eq 6a. First, the curves for higherAMPS-containing polyelectrolytes (PAMPS and DMF50) do not extrapolate to $Y_{\mathrm{c}}=0$ at zero salt. This observation indicates the presence of some effect that opposes a monotonic increase in polyelectrolyte-micelle attraction with decreasing I. A number of simulations ${ }^{53,57,58}$ have suggested the existence of loops in the bound polymer state, as shown for example, in Figure $6 \mathrm{I}$ and $\mathrm{J}$ for a quenched polyelectrolyte.

While the overall energy of complex formation is favorable, we may consider a hypothetical first step in which the polyelectrolyte (absent the micelle) attains its bound state configuration, an unfavorable process that becomes more so with increasing $\tau$ and decreasing $I$. Introducing the micelle into this system provides the stabilizing force, varying directly with $\tau$ and inversely with $I$. We can thus describe the two aspects that stabilize binding as "loop compliance" and "micelle affinity". Loop compliance decreases with $\tau$ and increases with $I$, while micelle affinity increases with $\tau$ and decreases with $I$. Maximum binding would occur with a polyelectrolyte that could form low 


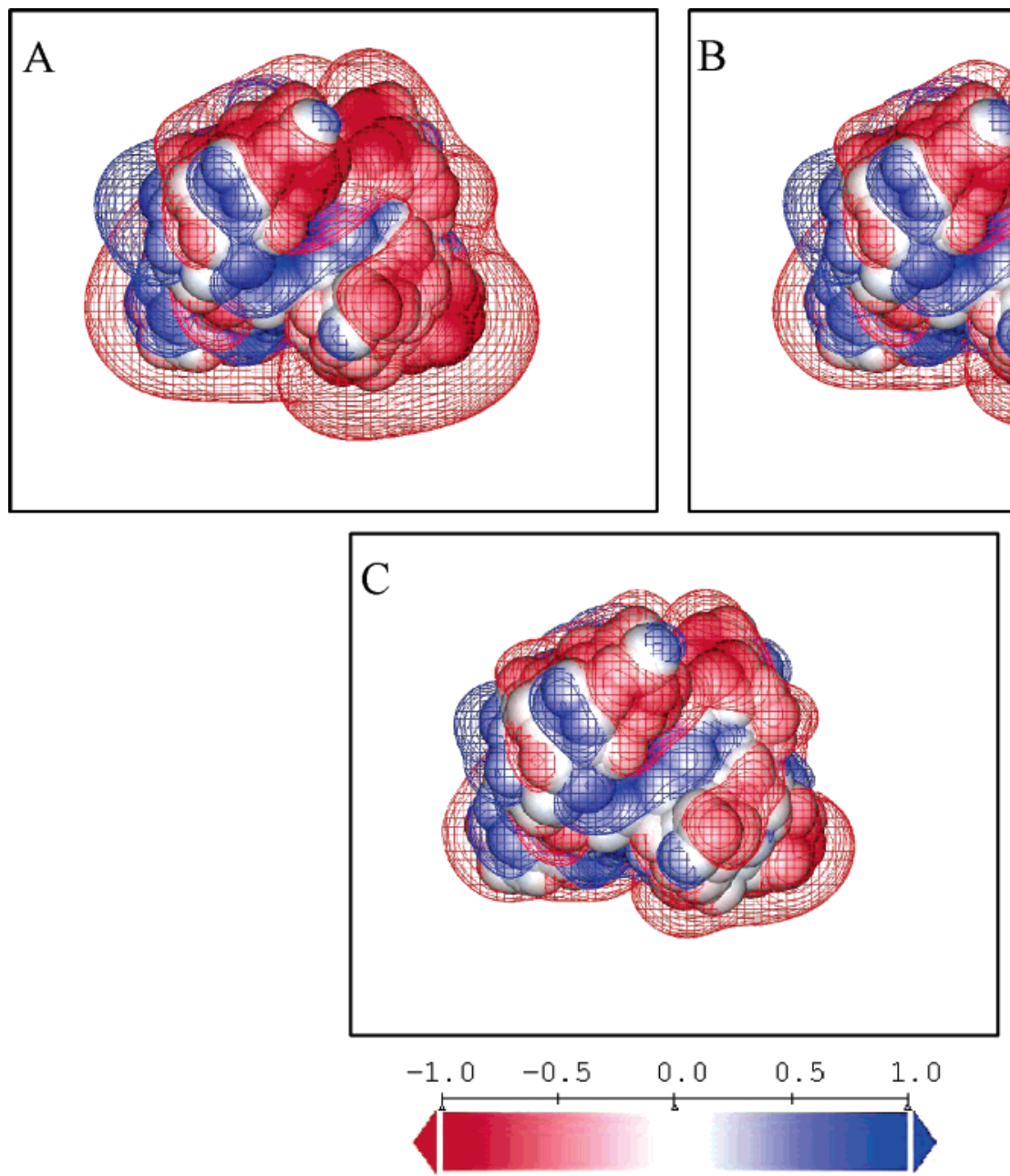

Figure 10. Electrostatic modeling of BSA under conditions of incipient binding with DMF25. The corresponding phase boundary points in Figure 9B are: $\mathrm{A}: \mathrm{pH}=6.8, I=0.10, Z=-6.9$; $\mathrm{B}: \mathrm{pH}=6.6, I=0.15, Z=-5.0$; $\mathrm{C}: \mathrm{pH}=6.3, I=0.25, Z=-2.9$. All images represent the same view. Potential surfaces: red $=-0.1 \mathrm{kT} / \mathrm{e}$ and blue $=0.1 \mathrm{kT} / \mathrm{e}$.

$\tau_{\text {eff }}$ loops and high $\tau_{\text {eff }}$ trains; all the copolyelectrolytes contain sequences that, to different degrees, approximate this arrangement. At low salt, where the Debye length $\kappa^{-1}$ is $30 \AA$, the loop compliance effect is dominant; hence the larger binding (compared to PAMPS or DMF50) for DMF20, which provides long runs of AAm. At high salt, the attractive force, of necessity more short-range than the repulsive one, becomes more important. This also explains the intermediate position of PAMPS (between DMF copolymers) at low salt $(I<0.2 \mathrm{M})$, as it provides less loop compliance but more short-range micelle affinity. When repulsions are screened above $I=0.36 \mathrm{M}$, where $\kappa^{-1}$ is on the order of $5 \AA$, PAMPS shows the strongest binding. This distance is also consistent with earlier estimates of the average distance between the micelle surface and a train of adsorbed polyion segments. ${ }^{15,59}$ This separation of loop formation and micelle binding is not rigorous, but whatever merits it displays suggest that it be included in refinements of eq 1 .

The second inconsistency with eq $6 \mathrm{a}$ is more problematic. The order of binding affinity is H2O38 > PAMPS > DMF20 $>$ DMF50, i.e., no simple correlation with average charge density. This also disagrees with results for similar polyanions binding to $\mathrm{N}, \mathrm{N}$-dimethyldodecylamine oxide (DMDAO) spherical micelles, reported by Feng et al., ${ }^{13}$ who obtained DMF25 $<$ DMF50 $\approx$ PAMPS. Binding to DMDAO micelles occurs at cationic surfactant mole fractions much lower than those found here for DTAB/TX-100, presumably because DTAB headgroups here are "buried" by TX-100 ${ }^{60}$ Bound polyelectrolyte segments in the current case reside further from the mean locus of charged surfactant headgroups, but the influence of this on the binding order is not evident at present.

Critical conditions for complex formation with proteins, shown in Figure 9B, differ in several ways from those for micelles. The prominent features are (1) the strong binding by DMF25, (2) the near convergence of phase boundaries for the other polymers, DMF50 displaying a small but significant enhancement of binding relative to PAMPS or $\mathrm{H} 2 \mathrm{O} 38$, (3) the remarkable linearity of the data for PAMPS and DMF50 at $I<$ $0.36 \mathrm{M}$ (very nearly a linear dependence of $Z_{\mathrm{c}}$ on the square root of ionic strength), and (4) the rather abrupt increase in slope at higher salt.

Protein affinity in the range $0.06<I<0.3 \mathrm{M}$, where $Z_{\mathrm{c}}<$ 0 , increases in the order PAMPS $\approx \mathrm{H} 2 \mathrm{O} 38<$ DMF50 $\ll$ DMF25. In contrast with the result for micelles, one observes for polymers synthesized in DMF an inverse relationship between the average polyelectrolyte linear charge density and protein binding. This higher level of complexity arises from the role of polyelectrolyte charge sequence distribution in protein binding. The optimal linear arrangements of polyelectrolyte charges are those that maximize attraction with the positive protein domain ("patch") yet minimize repulsion with the surrounding negative protein domain. DMF25, in particular, contains AMPS runs long enough ${ }^{29}$ to bind the positive protein domain, and these sequences statistically are readily bracketed 

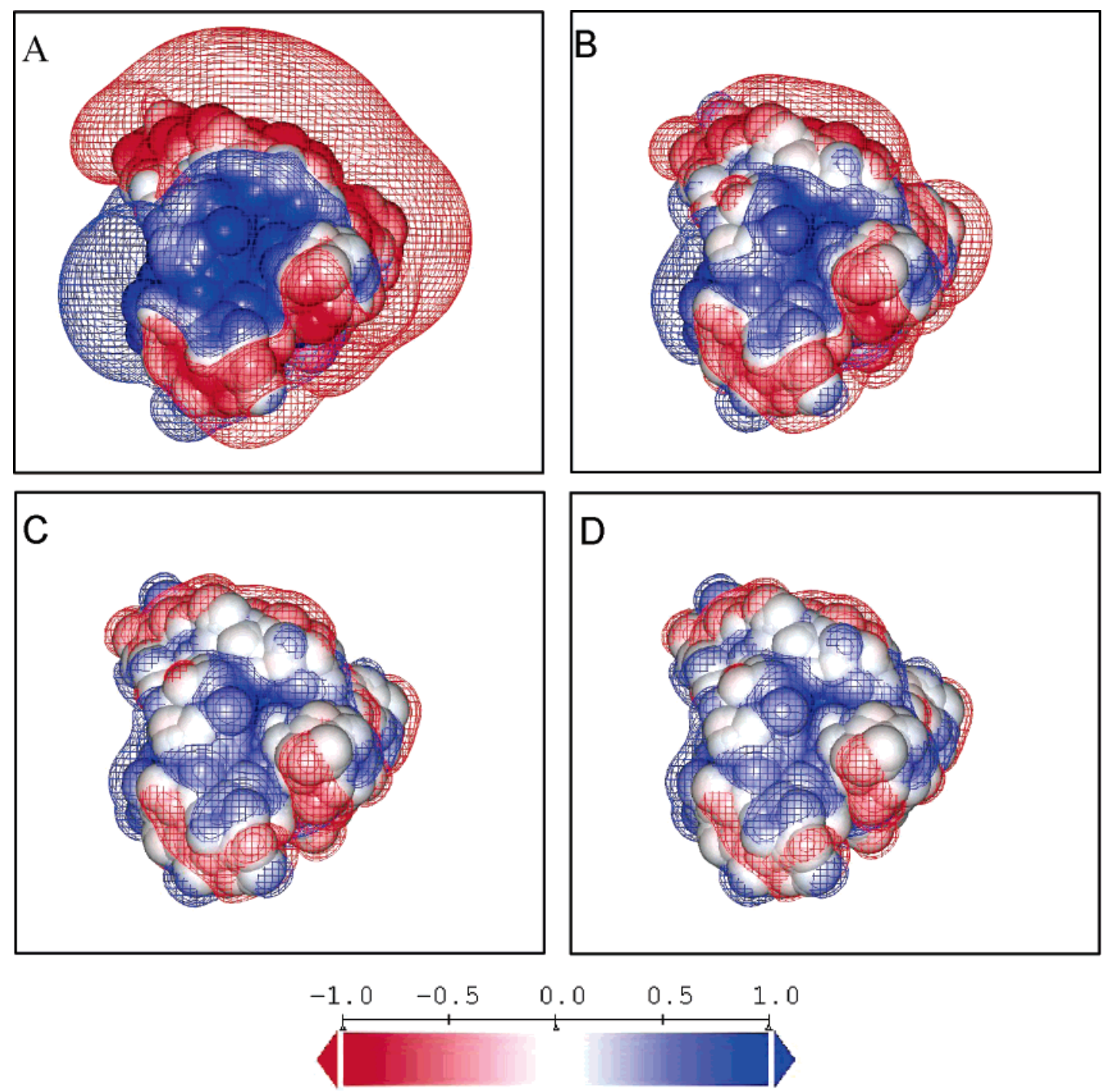

Figure 11. Electrostatic modeling of BSA under conditions of incipient complexation with PAMPS. The corresponding phase boundary points in Figure 9B are: $\mathrm{A}: \mathrm{pH}=6.2, I=0.05, Z=-3.1 ; \mathrm{B}: \mathrm{pH}=5.6, I=0.20, Z=2.4 ; \mathrm{C}: \mathrm{pH}=4.8, I=0.50, Z=10.2 ; \mathrm{D}: \mathrm{pH}=4.55, I=0.60$, $Z=14.9$. All images represent the same view. Potential surfaces: red $=-0.1 \mathrm{kT} / \mathrm{e}$ and blue $=0.1 \mathrm{kT} / \mathrm{e}$.

by runs of AAm that minimize repulsion from the negative protein domain.

In an attempt to rationalize the strongest binding of DMF25, electrostatic potential models of BSA were created at points corresponding to the onset of binding along the DMF25 phase boundary (see Figure 9B). In so doing, we sought to identify possible binding sites for DMF25 on the protein at the critical binding conditions. The criteria established for identifying the electrostatic binding site of a protein were previously put forward ${ }^{23}$ as follows: (1) the binding site location and size must be the same at all critical binding conditions along the phase boundary, (2) the binding domain must become appropriately larger or smaller upon $\mathrm{pH}$ adjustment, and (3) the mean potential $\psi$ on or within the binding domain should satisfy the relationship $z \psi \approx \mathrm{kT}$ (i.e., close to thermal energy), where $z$ is the number of polyelectrolyte charges binding cooperatively. For our purposes, we focus on the first two of these criteria. Figures 10 A-C, corresponding to $\mathrm{pH}$ and $I$ conditions at which DMF25 binds but the other polymers do not, show a small positive potential domain extending from the protein surface. Because the onset of binding is controlled by those sequences of polymers that are best suited to complex formation, we visualize a DMF25 sequence in which a run of AMPS units is bracketed by AAmrich runs that minimize repulsion from the nearby negative protein domains. From Figure 10, we estimate the size of the positive protein domain to be ca. $2 \mathrm{~nm}$. This domain can accommodate a sequence of approximately 7 AMPS units. The weaker binding of DMF50 indicates that the presence of such uncharged bracketing sequences is more important than the availability of longer runs of AMPS, and the even weaker binding of PAMPS confirms the importance of these repulsive effects. In fact, parts A-D of Figure 11, protein electrostatic potential models created at critical binding conditions for PAMPS, illustrate the need for a region of high positive potential of sufficient size so that bound PAMPS can avoid repulsion with nearby negative protein domains.

Examination of the data for DMF50 and PAMPS at $I<0.36$ $\mathrm{M}$ in Figure 9B shows a remarkably linear dependence for the critical protein net charge. This result at first suggests that polyelectrolyte binding is controlled by the net protein charge. However, the continuity of phase boundaries through $Z_{\mathrm{c}}=0$ along with polyelectrolyte binding "on the wrong side of" $\mathrm{p} I$ $(\mathrm{pH}>\mathrm{p} I)$ indicate that binding must instead be controlled by a local "patch" charge that may be opposite in sign to $Z$. To explain this, BSA was modeled at critical conditions along the PAMPS phase boundary, with the results shown in Figure 11AD. These models show a 4-nm positive potential domain ("patch") extending from the protein surface that is twice as 


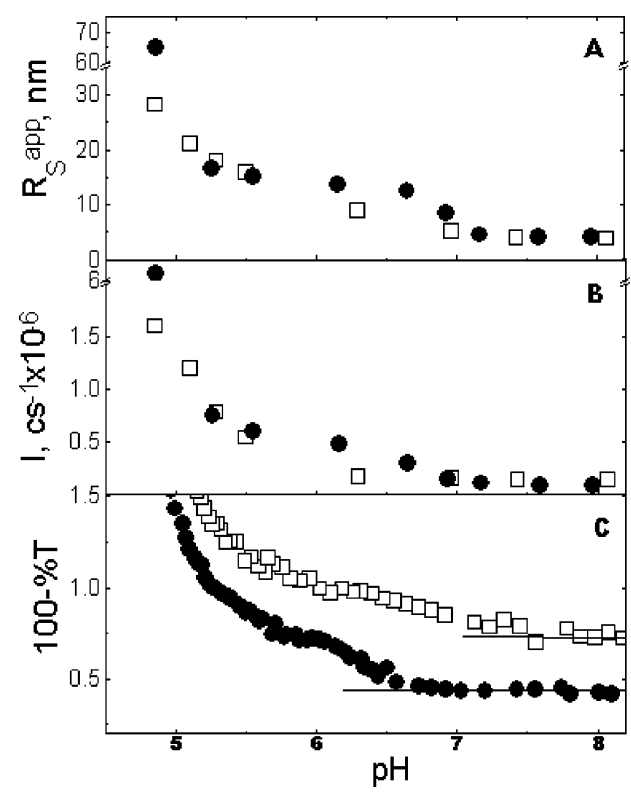

Figure 12. Comparison of turbidimetric titration and light scattering (DLS) results for PAMPS (filled circles) and DMF20 (open squares) Apparent mean Stokes radius determined by DLS (A), count rate (B), turbidity $(\mathrm{C})$. Lines are drawn to indicate the means of determining $\mathrm{pH}_{\mathrm{c}}$.

large as the potential domain observed at conditions corresponding to the onset of DMF25 binding. A similar positive binding domain was observed at all boundary conditions for $I$ $<360 \mathrm{mM}$; i.e., when positive charges are added to the protein in this ionic strength regime, corresponding to $-6<Z_{\mathrm{c}}<+4$, the patch becomes larger in size but retains the same geometry. In other words, all charges added to the protein in this range are added to the same positive patch, so the patch charge varies linearly with the net protein charge $\left(Z_{\text {net }}=Z_{\text {local }}+\right.$ constant $)$. This leads to the linear dependence of $Z_{\mathrm{c}}$ on $I$ at $I<0.36 \mathrm{M}$. Data for all polymers depart from this initial linear region and a second linear region, of steeper slope, is observed at $I>0.36$ M. Moving from the low $I$ regime to the high $I$ regime (from Figure $11 \mathrm{~A}$ and $\mathrm{B}$, to $\mathrm{C}$ and $\mathrm{D}$ ), we observe a striking difference in the protein charge distribution from a single, yet prominent, patch at low $I$, to a more heterogeneous charge distribution at high $I$. In this second regime, charges are added to the protein at sites outside of the polymer-binding domain identified at low I. These charges enhance the binding less than those that are concentrated in one region. As a result, we observe a decrease in the amount of additional salt required to desorb bound polyelectrolyte upon an incremental increase in $Z$. This results in an increase in the slope of Figure 9B, which changes by a factor of 2 between high and low salt regimes.

Characterization of Soluble Complexes. Structural information about the complexes of DMF20 and PAMPS with BSA may be derived from results from DLS measurements. Figure 12 shows the $\mathrm{pH}$ dependence of $(\mathrm{A})$ mean hydrodynamic radii, (B) count rate, and (C) turbidity in $10 \mathrm{mM} \mathrm{NaCl}$ for solutions containing $1.25 \mathrm{~g} / \mathrm{L}$ of BSA and $0.25 \mathrm{~g} / \mathrm{L}$ of polymer. All three plots show a region "I" of no interaction at high $\mathrm{pH}\left(>\mathrm{pH}_{\mathrm{c}}\right)$ in which no $\mathrm{pH}$ dependence is seen, a region "III" in which scattering and apparent size increase rapidly $\left(\mathrm{pH}<\mathrm{pH}_{\phi}\right)$, signaling phase separation, and an intermediate region " $\mathrm{II}$ " $\left(\mathrm{pH}_{\phi}\right.$ $<\mathrm{pH}<\mathrm{pH}_{\mathrm{c}}$ ) of soluble complexes. The values of $\mathrm{pH}_{\mathrm{c}}$ for PAMPS and DMF20 are 6.7 and 7.4, respectively; the corresponding values for $\mathrm{pH}_{\phi}$ are 5.1 and 5.3, respectively.

At $\mathrm{pH}>\mathrm{pH}_{\mathrm{c}}$ (region $\mathrm{I}$ ), the turbidity and the size are nearly constant with respect to $\mathrm{pH}$, indicating that no interaction exists between polyelectrolytes and BSA. The concentration and refractive index increment of the polyelectrolytes are both low relative to BSA, and the small values of $R_{\mathrm{S}}$ app arise mainly from protein scattering. In this region, Coulombic repulsion between negatively charged protein and negatively charged polyelectrolytes prohibits complex formation. The formation of soluble complexes at $\mathrm{pH}_{\phi}<\mathrm{pH}<\mathrm{pH}_{\mathrm{c}}$ (region II) is indicated by an increase in the turbidity, the size $\left(R_{\mathrm{S}}\right)$, and the average count rate, but these increases, which are gradual for DMF20, exhibit plateaus centered around $\mathrm{pH} 6$ for PAMPS. The region of soluble complexation (II) is greater for DMF20 than for PAMPS. For the fully charged PAMPS, repulsive effects may limit the number of proteins bound per polymer chain $(n)$. At $\mathrm{pH} \approx 5$, complexes approach electrical neutrality and higher-order aggregation commences, leading to phase separation. DMF20, in contrast to PAMPS, where all protein-binding sites are similar, can contain a heterogeneous and essentially limitless variety of protein-binding sites of different protein affinities, all of which are characterized by an AMPS-rich sequence bracketed by an AMPS-poor sequence. Thus, progressive binding of protein is continuous with decreasing $\mathrm{pH}$. This heterogeneity blurs the transition from region II to region III for the copolymer.

\section{Conclusions}

The binding affinity of anionic polyelectrolytes for a protein (BSA) or for an oppositely charged micelle is strongly influenced by three polyelectrolyte structural parameters: chain flexibility, charge mobility, and charge density. In general, the first two promote binding, as observed by an expansion of the range of solution conditions (e.g., $\mathrm{pH}$ and ionic strength $I$ ) in which complex formation occurs. The influence of polymer charge density is more complex and depends on colloid charge heterogeneity, the conformation of the adsorbed polymer, and I. Comparisons among different polyanions are facilitated by expressing the conditions for complexation as $\sigma=K I^{a}$, where the experimental analogue of the surface charge density $\sigma$ is the net charge of the protein or the mole fraction of ionic surfactant of the micelle. The value of $a$ is near 0.5 for BSA and near unity for micelles. More subtle effects seen for protein binding were attributed to charge heterogeneity, which introduces repulsive forces between polyanions and protein negative domains, and also alters the nature of the polyanion-binding site. However, the surprising consistency of this site over a wide range of $I$ gives rise to linearity between $I$ and the net protein charge at the onset of binding. Repulsive effects for micelle binding, on the other hand, appear to arise from intrapolymer interactions within bound loops. Charge complementarity may play a special role in protein binding, with the surprising consequence of high protein affinity for a copolymer with a low content of ionic residues.

Acknowledgment. Support from NSF CHE-0345382 is acknowledged.

\section{References and Notes}

(1) Lander, A. D. Matrix Biol. 1998, 17, 465-472.

(2) Seyfried, N. T.; McVey, G. F.; Almond, A.; Mahoney, D. J.; Dudhia, J.; Day, A. J. J. Biol. Chem. 2005, 280, 5435-5448.

(3) Wang, Y.; Dubin, P. L. Interfacial Phenomena in Chromatography. In Surf. Sci. Ser. 1999, 80, 311-327,

(4) Strege, M. A.; Dubin, P. L.; West, J. S.; Flinta, C. D. In Protein Purification: Molecular Mechanics of Large-Scale Processes; ACS Symposium Series 427; American Chemical Society: Washington, DC, 1990, pp 66-79. 
(5) Decher, G.; Eckle, M.; Schmitt, J.; Struth, B. Curr. Opin. Colloid Interface Sci. 1998, 3, 32-39.

(6) Ivinova, O. N.; Izumrudov, V. A.; Muronetz, V. I.; Galaev, I. Y.; Mattiasson, B. Macromol. Biosci. 2003, 3, 210-215.

(7) Cooper, C. L.; Dubin, P. L.; Kayitmazer, A. B.; Turksen, S. Curr. Opin. Colloid Interface Sci. 2005, 10, 52-78.

(8) Weigel, F. W. J. Phys. A.: Math. Gen. 1977, 10, 299.

(9) Muthukumar, M. J. Chem. Phys. 1987, 86, 7230-7235.

(10) von Goeler, F.; Muthukumar, M. J. Chem. Phys. 1994, 100, 77967803.

(11) Evers, O. A.; Fleer, G. J.; Scheutjens, J. M. H. M.; Lyklema, J. J. Colloid Interface Sci. 1986, 111, 446-454.

(12) Zhang, H.; Ohbu, K.; Dubin, P. L. Langmuir 2000, 16, 9082-9086.

(13) Feng, X. H.; Dubin, P. L.; Zhang, H. W.; Kirton, G. F.; Bahadur, P.; Parotte, J. Macromolecules 2001, 34, 6373-6379.

(14) Zhang, H.; Dubin, P. L.; Ray, J.; Manning, G. S.; Moorefield, C. N.; Newkome, G. R. J. Phys. Chem. B 1999, 103, 2347-2354.

(15) Dubin, P. L.; Thè, S. S.; McQuigg, D. W.; Chew, C. H.; Gan, L. M. Langmuir 1989, 5, 89-95.

(16) Dubin, P. L.; Rigsbee, D. R.; McQuigg, D. W. J. Colloid Interface Sci. 1985, 105, 509-515.

(17) Thé, S. M.S. Thesis, Purdue University, Indianapolis, IN, 1989.

(18) Zhang, H.; Dubin, P. L.; Spindler, R.; Tomalia, D. Ber. BunsenGes. Phys. Chem. 1996, 100, 923-928.

(19) Kayitmazer, A. B.; Seyrek, E.; Dubin, P. L.; Staggemeier, B. A. J. Phys. Chem. B 2003, 107, 8158-8165.

(20) McQuigg, D. W.; Kaplan, J. I.; Dubin, P. L. J. Phys. Chem. 1992, 96, 1973-1978.

(21) Loeb, A. L.; Overbeek, J. T. G.; Wiersema, P. H. The Electrical Double Layer Around a Spherical Colloid Particle; Computation of the Potential, Charge Density, and Free Energy of the Electrical Double Layer around a Spherical Colloid Particle; MIT Press: Cambridge, MA, 1961.

(22) Seyrek, E.; Dubin, P. L.; Tribet, C.; Gamble, E. A. Biomacromolecules 2003, 4, 273-282.

(23) Grymonpré, K. R.; Staggemeier, B. A.; Dubin, P. L.; Mattison, K. W. Biomacromolecules 2001, 2, 422-429.

(24) Park, J. M.; Muhoberac, B. B.; Dubin, P. L.; Xia, J. Macromolecules 1992, 25, 290-295.

(25) Mattison, K. W.; Dubin, P. L.; Brittain, I. J. J. Phys. Chem. B 1998 , $102,3830-3836$.

(26) Kong, C. Y.; Muthukumar, M. J. Chem. Phys. 1998, 109, 15221527.

(27) Wallin, T.; Linse, P. Langmuir 1996, 12, 305-314.

(28) Yoshida, K.; Dubin, P. L. Colloids Surf., A 1999, 147, 161-167.

(29) Hattori, T.; Kimura, K.; Seyrek, E.; Dubin, P. L. Anal. Biochem. 2001, 295, 158-167.

(30) Gao, J. Y.; Dubin, P. L.; Sato, T.; Morishima, Y. J. Chromatogr., A 1997, 766, 233-236.

(31) Xia, J.; Dubin, P. L.; Dautzenberg, H. Langmuir 1993, 9, 20152019.

(32) Tsuboi, A. I.; Hirata, M.; Dubin, P. L.; Kokufuta, E. Langmuir 1996, $12,6295-6303$.
(33) Bohidar, H.; Dubin, P. L.; Majhi, P.; Tribet, C.; Jaeger, W. Biomacromolecules 2005, 6, 1573-1585.

(34) Verwey, E. J. W.; Overbeek, J. Th. G. Theory of the Stability of Lyophobic Colloids; Elsevier: Amsterdam, 1962.

(35) Metropolis, N.; Rosenbluth, A. W.; Rosenbluth, M. N.; Teller, A. H.; Teller, E. J. Chem. Phys. 1953, 21, 1087-1092.

(36) Ullner, M.; Joensson, B.; Widmark, P. O. J. Chem. Phys. 1994, 100, 3365-3366.

(37) Reed, C. E.; Reed, W. F. J. Chem. Phys. 1991, 94, 8479-8486.

(38) Tanford, C.; Kirkwood, J. G. J. Am. Chem. Soc. 1957, 79, 53335339.

(39) Zhang, B.; Kirton, G. F.; Dubin, P. L. Langmuir 2002, 18, 46054609

(40) Kirton, G. F.; McQuigg, D. W.; Dubin, P. L. Abstracts of Papers, 36th Central Regional Meeting of the American Chemical Society, Indianapolis, IN, June 2-4, 2004; American Chemical Society, Washington, DC, 2004.

(41) Mandel, M. Eur. Polym. J. 1970, 6, 807-822.

(42) Cesaro, A.; Ciana, A.; Delben, F.; Manzini, G.; Paoletti, S. Biopolymers 1982, 21, 431-449.

(43) Cleland, R. L.; Wang, J. L.; Detweiler, D. M. Macromolecules 1982, $15,386-395$.

(44) Staggemeier, B. M.S. Thesis, Purdue University, Indianapolis, IN, 1999

(45) Tricot, M. Macromolecules 1984, 17, 1698-1704.

(46) Harding, S. E.; Berth, G.; Ball, A.; Mitchell, J. R.; Garcia de la Torre, J. Carbohydr. Polym. 1991, 16, 1-15.

(47) Cros, S.; Garnier, C.; Axelos, M. A. V.; Imberty, A.; Perez, S. Biopolymers 1996, 39, 339-352.

(48) Hayashi, K.; Tsutsumi, K.; Nakajima, F.; Norisuye, T.; Teramoto, A. Macromolecules 1995, 28, 3824-3830.

(49) Comper, W. D.; Laurent, T. C. Physiol. Rev. 1978, 58, 255-315.

(50) Reed, W. F.; Ghosh, S.; Medjahdi, G.; Francois, J. Macromolecules 1991, 24, 6189-6198.

(51) Stubenrauch, C.; Albouy, P.-A.; v. Klitzing, R.; Langevin, D. Langmuir 2000, 16, 3206-3213.

(52) Miyamoto, S. Macromol. Chem. Phys. 1981, 182, 559-568.

(53) Ulrich, S.; Laguecir, A.; Stoll, S. Macromolecules 2005, 38, 89398949.

(54) Stoll, S.; Chodanowski, P. Macromolecules 2002, 35, 9556-9562.

(55) Morishima, Y. personal communication.

(56) McCormick, C. L.; Chen, G. S. J. Polym. Sci., Polym. Chem. Ed 1982, 20, 817-838.

(57) Chodanowski, P.; Stoll, S. Macromolecules 2001, 34, 2320-2328

(58) Chodanowski, P.; Stoll, S. J. Chem. Phys. 2001, 115, 4951-4960.

(59) Hattori, T.; Hallberg, R.; Dubin, P. L. Langmuir 2000, 16, 97389743.

(60) Zhang, H.; Li, Y.; Dubin, P. L.; Kato, T. J. Colloid Interface Sci. 1996, $183,546-551$

BM050592J 